1 Fundação Oswaldo Cruz (Fiocruz), Instituto René Rachou (IRR) - Belo Horizonte (MG), Brasil. rhavena.santos@gmail.com

2 Fundação Oswaldo Cruz (Fiocruz), Escola Nacional de Saúde Pública Sergio Arouca (Ensp) - Rio Janeiro (RJ), Brasil.

\section{Vigilância em saúde e desastres de origem natural: uma revisão da literatura}

\author{
Health surveillance and natural disasters: a literature review
}

Rhavena Santos ${ }^{\mathbf{1}}$, Júlia Alves Menezes ${ }^{\mathbf{1}}$, Carina Margonari de Souza', Ulisses Confalonieri', Carlos
Machado de Freitas

DOI: $10.1590 / 0103-11042020 E 222$

\begin{abstract}
RESUMO As projeções climáticas para o final do século XXI sugerem a intensificação de eventos extremos que podem causar danos diretos e indiretos à saúde pública e impactar, em diferentes proporções, as populações vulneráveis. Nesse âmbito, destaca-se a vigilância em saúde por suas relevantes ações em todas as etapas de gestão de risco dos desastres. O objetivo deste artigo foi realizar uma revisão da literatura científica sobre a vigilância em saúde e desastres de origem natural, com intuito de descrever as principais abordagens desses temas. Observou-se que as publicações têm como assunto central os efeitos dos desastres sobre a saúde; as ações do setor saúde em desastres, o sistema/fluxo de informação em saúde e as condições de vulnerabilidade aos desastres. Os dois primeiros tópicos são mais prevalentes na literatura e permitem o compartilhamento das lições aprendidas ao longo dos anos. Embora em menor número, os dois últimos temas apresentam discussões relevantes para a temática da gestão de risco de desastres e têm muito a contribuir para o avanço da cultura de risco no País, para uma melhor compreensão da realidade e para a resiliência tanto populacional quanto institucional. Nesse sentido, ressalta-se a importância de mais estudos sobre essas temáticas.
\end{abstract}

PALAVRAS-CHAVE Vigilância em saúde pública. Desastres naturais. Saúde pública. Vulnerabilidade a desastres. Sistemas de informação.

ABSTRACT Climate projections for the end of the 21st century suggest the intensification of extreme events that can cause direct and indirect damage to public health and impact differently on vulnerable populations. In this context, health surveillance stands out for its relevant actions in all stages of disaster risk management. The aim of this paper was to review the scientific literature on health surveillance and natural disasters in a global context, in order to describe the main approaches to such themes. Publications tend to focus on the health effects of disasters; health sector actions in disasters, the health information system/flow, and conditions of vulnerability and disasters. The first two topics are more prevalent in the literature and allow the sharing of lessons learned over the years. Although in smaller numbers, the last two themes present discussions relevant to the theme of disaster risk management and have much to contribute to the advancement of risk culture in the country, for a better understanding of the reality and for the resilience of both the population and institutions. In this sense, the importance of further studies on these themes is emphasized.

KEYWORDS Public health surveillance. Natural disasters. Public health. Disaster vulnerability. Information Systems. 


\section{Introdução}

As projeções climáticas para o final do século XXI sugerem o aumento das temperaturas médias do planeta e a intensificação de eventos extremos (por exemplo, secas, tempestades, furacões), que podem impactar os ecossistemas vulneráveis à mudança do clima em diferentes proporções ${ }^{\mathbf{1 , 2}}$.

Sob o ponto de vista da saúde pública, essas mudanças causam impactos diretos (mortos, feridos) e indiretos (migrações, insegurança alimentar) às populações. Dados do Atlas Brasileiro de Desastres Naturais evidenciam que, no Brasil, entre os anos de 1991 e 2012, foram registrados 126.926 .656 danos humanos por desastres, sendo 3.448 óbitos $^{3}$.

A temática de desastres ganhou destaque mundial, principalmente na década de 1990, reconhecida como Década Internacional para Redução de Desastres Naturais pela Assembleia das Nações Unidas ${ }^{4}$. Na segunda metade dos anos 1990, o termo gestão de risco de desastres foi evidenciado ao abarcar a noção de risco potencial de dano e perdas. Para Narváez et al.5(33), o termos gestão de risco de desastre pode ser entendido como:

[...] processo social cujo objetivo final é a previsão, a redução e o controle permanente dos fatores de risco de desastres na sociedade, alinhados e integrados à consecução de diretrizes de desenvolvimento humano, econômico, ambiental e territorial sustentáveis.

$\mathrm{O}$ risco é resultante da interação entre a ameaça (natural ou antrópica) e a vulnerabilidade presente em uma localidade, dessa forma, o reconhecimento destas, quando associadas a características locais, como, condições sociais, de saúde, econômicas, ambientais, culturais e demográficas, auxilia a gestão do risco de desastres nas suas três etapas, a saber: redução do risco; manejo e recuperação ${ }^{6}$.

Em âmbito mundial, a discussão sobre gestão do risco de desastres vem sendo abordada ao longo dos anos por eventos internacionais, como as conferências mundiais sobre desastres em Yokohama (1994), Kobe (2005) e Sendai (2015). Nesta última, os objetivos relacionados com a noção do risco são claros ao abordarem a necessidade tanto de prevenção de novos riscos quanto de redução dos existentes, estabelecendo prioridades que abrangem: compreensão do risco de desastre; fortalecimento da governança para gerenciamento; redução do risco e resiliência ${ }^{7}$.

Além disso, o Marco de Sendai está de acordo com a Agenda 2030 para o desenvolvimento sustentável, que define áreas para o avanço do desenvolvimento considerando as dimensões econômica, social e ambiental. Estabelece também, entre outras prioridades, a erradicação da pobreza, a redução das desigualdades, ações contra mudança do clima global, saúde e bem-estar, além de reforçar a natureza universal e colaborativa desse processo ${ }^{8}$. Nessa perspectiva, a correspondência entre esses acordos internacionais aliada aos determinantes sociais da saúde têm muito a contribuir para melhora da saúde pública.

Diante de um desastre, o setor saúde desenvolve ações com o intuito de garantir a prevenção, promoção, proteção, recuperação e reabilitação da saúde ${ }^{6}$. Nesse âmbito, destaca-se ainda a vigilância em saúde, por seu potencial de contribuição no entendimento dos desastres. Ela atua na vigilância, prevenção e controle de doenças transmissíveis, vigilância de fatores de risco relacionados com doenças crônicas não transmissíveis, na saúde ambiental e do trabalhador, bem como na análise da situação de saúde 9 . Além disso, engloba um processo "contínuo e sistemático de coleta, consolidação, disseminação de dados sobre eventos relacionados à saúde"9(1), que tem muito a contribuir para o diagnóstico da realidade, que precede à elaboração e implementação de medidas para prevenção e controle de riscos, agravos e doenças9,10.

Em desastres, ações da vigilância em saúde contribuem para evitar ou mitigar a exposição das populações e profissionais de saúde aos riscos associados aos desastres, para reduzir o número de doenças e agravos decorrentes desses eventos e para preparar o setor saúde para prestar uma assistência rápida e de 
qualidade na ocorrência deles ${ }^{6}$. A utilização dos dados produzidos pela vigilância em saúde é importante na quantificação dos impactos decorrentes de desastres, no acompanhamento cronológico do número de eventos e danos causados à população (figura 1).

Figura 1. Ações da vigilância em saúde na gestão do risco de desastres

- Conhecimento do perfil de morbidade e mortalidade da população;

- Articulação intra e interinstitucional;

- Participação em ações

socioeducativas;

- Participação na elaboração do

plano de emergências;

- Capacitação de pessoal;

- Conhecimento de fatores de risco e

populações vulneráveis:

- Ações de redução de

vulnerabilidades que envolvem a

pobreza extrema; o deficit

habitacional; a infraestrutura urbana

inadequada; a degradação ambiental

e de áreas frágeis; o adensamento

populacional.
- Distribuição de kit de medicamentos

e insumos estratégicos;

- Controle epidemiológico de

doenças;

- Participar da organização de

abrigos;

- Registro e avaliação dos danos a

saúde e instalações físicas;

- Comunicação e tomada de decisões

intra e interinstitucional.
- Controle de doenças em médio e

longo prazo;

- Avaliação do perfil de

morbimortalidade;

- Avaliação de novas necessidades de

saúde e perfil de vulnerabilidade da

população;

- Comunicação e tomada de decisões

intra e interinstitucional.
Assim, esta revisão justifica-se em um cenário crescente de ocorrências e intensidade dos desastres de origem natural, em que as ações de vigilância em saúde são relevantes em todas as etapas da gestão de risco de um desastre e podem contribuir para a diminuição dos impactos desses eventos sobre a população.

Dessa forma, o objetivo deste artigo é realizar uma revisão sistemática de artigos que abordem a relação entre a vigilância em saúde e os desastres de origem natural com intuito de descrever as principais abordagens desses temas e, dessa forma, contribuir para reflexões sobre as práticas da vigilância em saúde e desastres no Brasil.

\section{Material e métodos}

Uma pesquisa da literatura foi realizada entre fevereiro e maio de 2019 para identificar trabalhos revisados por pares que tratassem do tema da saúde pública e desastres. Foram utilizadas três bases de dados: PubMed, Portal de periódicos da Coordenação de Aperfeiçoamento de Pessoal de Nível Superior (Capes) e Portal da Biblioteca Virtual em Saúde (BVS) - Preparação e resposta a desastres. A primeira por ser considera uma base abrangente e, ao mesmo tempo, específica para a saúde; a segunda por ser o modelo de biblioteca financiado pelo governo brasileiro e a última por disponibilizar informações e evidências científicas relacionadas com desastres.

Não houve restrição quanto à abrangência dos estudos, podendo os artigos ser nacionais e internacionais, independentemente do ano da publicação, porém, o idioma foi restrito ao inglês, ao português ou ao espanhol. Embora exista considerável literatura adicional distribuída por vários relatórios institucionais, livros e monografias, essas publicações não foram incluídas nesta revisão.

Para busca na base de dados PubMed, foram usados os descritores 'Public Health Surveillance' e 'Natural Disasters', relacionados por meio do operador booleano $A N D$. As buscas nas bases de 
dados dos Portais Capes e BVS foram realizadas com os Descritores em Ciências da Saúde (DeCS) 'Vigilância' e 'Desastres Naturais'. Optou-se por não utilizar o descritor 'Vigilância em Saúde pública', pois o termo restringiu a busca e não retornou resultados. O mesmo operador booleano foi utilizado nos Portais Capes (em que se optou pela busca por assunto) e BVS.

Os artigos selecionados seguiram para a etapa de leitura dos títulos e depois para leitura dos resumos; aqueles que não descreviam situações ou análises que abordassem o assunto desastres de origem natural foram excluídos. Não foi obrigatório constar o tema saúde na leitura do título ou dos resumos. Esse tema é frequentemente abordado dentro de um amplo espectro de consequências dos desastres e engloba variados determinantes sociais (por exemplo, condições econômicas, sociais e demográficas) que podem estar associados ao risco de desastres.

Após a leitura na íntegra, foram selecionados os artigos que relataram situações de desastres específicas (como o furacão Sandy, a seca na Amazônia brasileira), ou desastres (isto é, qualquer evento caracterizado como desastre de origem natural) e que estavam relacionados com a saúde e/ou com seus determinantes sociais. Ao final, um total de 43 artigos foram selecionados para análise (figura 2). A qualidade dos estudos selecionados não foi avaliada formalmente devido a diferenças: i) nas exposições aos desastres; e ii) na atuação e conformação dos diferentes sistemas de saúde.

Figura 2. Seleção dos artigos nas bases de dados PubMed, Capes e BVS

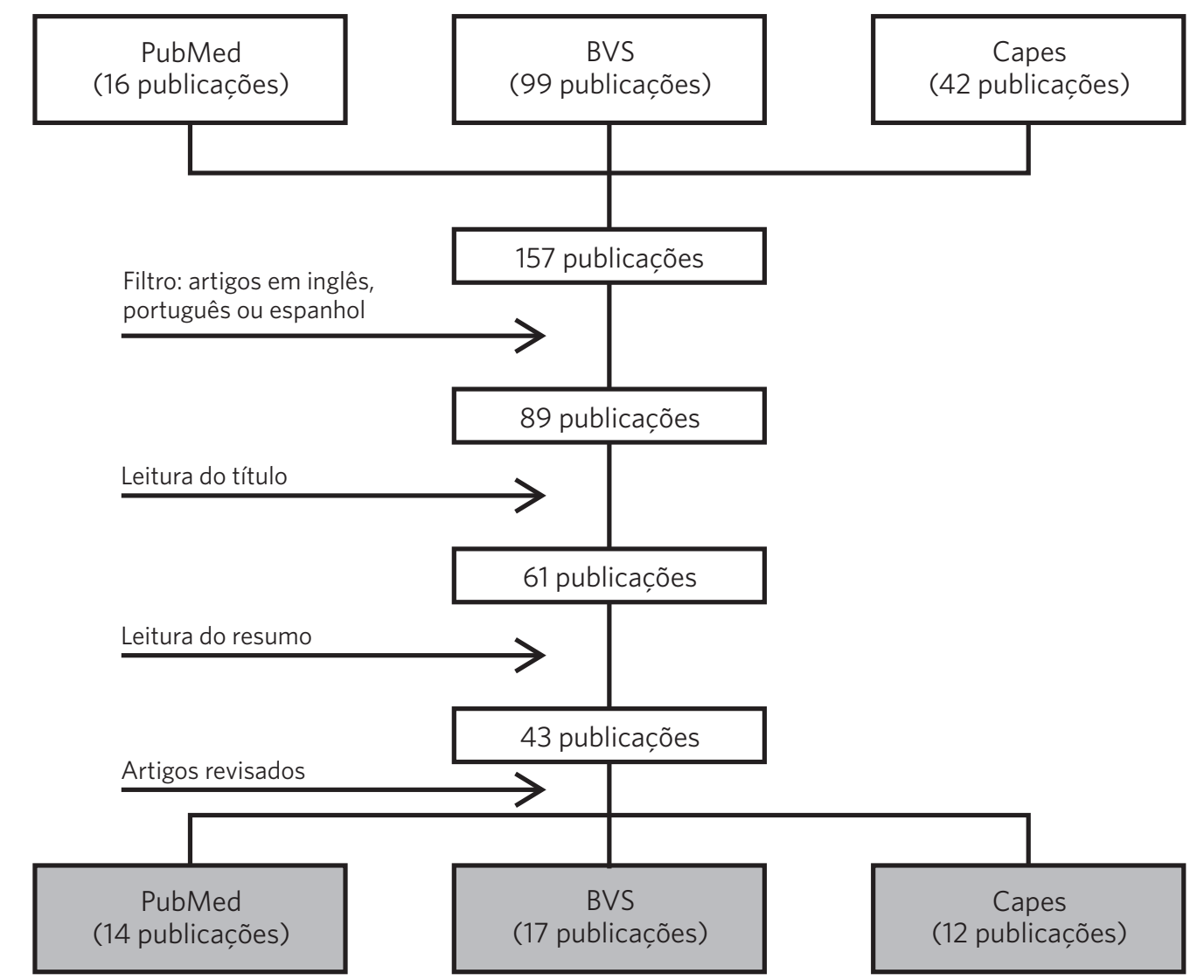

Fonte: Elaboração própria. 
A próxima etapa consistiu no fichamento e análise segundo a sistematização de informações. Esta foi realizada em uma matriz construída no Microsoft Excel $^{\circledR} 2010$ contendo o ano da publicação, o eixo temático do artigo e síntese de informações que pudessem auxiliar na compreensão do das abordagens sobre vigilância em saúde em desastres. São elas:

- Evento: em que se avaliou(aram) o(s) acontecimento(s) considerado(s) ameaça para população;

- Exposição: avaliaram-se características/ situações que permitissem estabelecer possíveis relações entre a população, os eventos disparadores e os impactos desses sobre o ambiente;

- Vulnerabilidades: estados de precárias condições de vida e proteção social decorrentes de processos sociais e mudanças ambientais que tornam as populações mais propensas a sofrer danos;

- Ações do setor saúde: principais ações realizadas pelo setor saúde para gestão de risco de um desastre, descritas como sendo de responsabilidade individual ou conjunta com outros setores;

- Efeito sobre a saúde: impactos diretos e indiretos dos desastres sobre a saúde das populações;

- Sistemas/fluxo de informação utilizados nos desastres: conjunto de arquivos, procedimentos e equipamentos utilizados para o armazenamento, manipulação, recuperação e divulgação de informações em desastres, independentemente da natureza física ou digital.

\section{Resultados e discussão}

Os textos foram organizados segundo seu tema central de discussão nas categorias: efeitos dos desastres sobre a saúde; ações do setor saúde em desastres; sistemas/fluxo de informação em saúde; condições de vulnerabilidade e desastres. Apenas um artigo não se encaixou nessas categorias por abordar o perfil das publicações sobre desastres na base de dados especializada em desastres BVS ${ }^{11}$. Alguns artigos se encaixaram em mais de uma categoria conforme quadro 1.

Quadro 1. Ações do setor saúde, sistema/fluxo de informações, condições de vulnerabilidade e efeitos dos desastres sobre a saúde das populações

\begin{tabular}{|c|c|}
\hline $\begin{array}{l}\text { Autor (ano) segundo categoria 'Efeitos dos desastres sobre a } \\
\text { saúde' }\end{array}$ & $\begin{array}{l}\text { rincipais 'Efeitos dos desastres sobre a saúde' descritos por artigos desta } \\
\text { ategoria }\end{array}$ \\
\hline $\begin{array}{l}\text { De Ville de Goyet, C. (1980); Ahearn, F. (1984); Hospedales, J. et } \\
\text { al., (1990); Duff, E. M. W. et al., (1991); Roces, M.C. et al., (1992); } \\
\text { Armenian, H. K.; Noji, E. K. \& Oganesian, A. P. (1992); Lima, B. R. et } \\
\text { al., (1992); Duff, E. M. \& Cooper, E. S. (1992); Duff, E. M. \& Cooper, } \\
\text { E. S. (1994); Malilay, J. et al., (1996); OPAS (1998); Balluz, L. et al., } \\
\text { (2001); Williams, E. W. et al., (2005); Raguenaud, M., et al. (2012); } \\
\text { R. Goulding, R. \& Jayasuriya, N.; Horan, E. (2012); Apisarnthana- } \\
\text { raka, Anucha; Warrenb, David K. \& Mayhall, Clovus Glen (2013); } \\
\text { Smith, L. T. et al., (2014); Guimarães, R. M. et al., (2014); Freitas, } \\
\text { C. M. et al., (2014); Pirard, P. et al., (2015); Shrestha, A. C. et al., } \\
\text { (2016); Sahni, V. et al., (2016); Ishiki, A. et al., (2016); Grigoletto, J. } \\
\text { C. et al., (2016); Rufino, R. et al., (2016); Sena, A. et al., (2016); Al- } \\
\text { pino, T. A., Sena, A. R. M. D., \& Freitas, C. M. D. (2016); Takahashi, } \\
\text { A. et al., (2017) }\end{array}$ & $\begin{array}{l}\text { - Introdução de novos patógenos no ambiente; } \\
\text { - Intensificação de transmissão de patógenos locais; } \\
\text { - Doenças de transmissão hídrica e alimentar, doenças transmitidas por vetores, } \\
\text { zoonoses; } \\
\text { - Doenças respiratórias; } \\
\text { - Doenças infecciosas fúngicas / respiratórias / dermatológicas / parasitárias / } \\
\text { alérgicas; } \\
\text { - Lesões e mortes; } \\
\text { - Doenças psicossociais, comportamentais, cardiovasculares; } \\
\text { - Desnutrição e desidratação; } \\
\text { - Intensificação de doenças crônicas; } \\
\text { - Violência física. }\end{array}$ \\
\hline
\end{tabular}


Quadro 1. (cont.)

Autor (ano) segundo categoria 'Acões do setor saúde em desastres'

Anon (1983); Hospedales, J. et al., (1990); Iversson, L. B. (1991); Balluz, L. et al. , (2001); Pérez, L. J. (2001); Williams, E. W. et al., (2005); Raguenaud, M. et al., (2012); Apisarnthanaraka, Anucha; Warrenb, David K. \& Mayhall, Clovus Glen (2013); Ridpath, A. D. et al. , (2015); Sahni, V. et al., (2016); Grigoletto, J. C. et al., (2016); Boyd, A. T. et al., (2017); Lemoine, J. F. et al., (2017)
Principais 'Ações do setor saúde em desastres' descritas por artigos desta categoria

- Ações de vigilância em curto, médio e longo prazo (p. e. levantamento global da área onde ocorreu o desastre; notificação de casos ou óbitos suspeitos de doenças infecciosas e isolamento/tratamento de casos; cuidados sanitários com os cadáveres; entrosamento com o laboratório de saúde pública; reuniões diárias de equipe; estabelecimento rápido das ações profiláticas que abrangem medidas de imunoproteção das pessoas e de saneamento ambiental; levantamento de equipamentos, medicamentos e produtos imunoprofiláticos disponíveis e estruturação de um sistema de divulgação e de recepção do que é realmente necessário, monitorização da qualidade da água, vigilância sobre impactos na saúde etc.);

- Elaboração de planos de contingência e resposta a emergências;

- Limpeza e descontaminação dos ambientes;

- Acompanhamento da saúde mental em curto, médio e longo prazo;

- Solicitação de auxílio internacional;

- Discussões sobre planos de saúde em desastres;

- Oficinas para discussão sobre comunicação de saúde entre as unidades de saúde em casos de desastres;

- Acordos internacionais em situações de desastres.

Autor (ano) segundo categoria 'Condições de vulnerabilidade e Principais 'Condições de vulnerabilidade e desastres' descritas por artigos

desastres' desta categoria

Freitas, C. M. et al., (2012); Freire, N. C. F., Bonfim, C. V. D., \& Na-

- Vulnerabilidade social, ambiental, institucional, organizacional, climática, econômica e demográfica.

Autor (ano) segundo categoria 'Sistemas/fluxo de informação

\section{em saúde'}

Principais 'Sistemas/fluxo de informação em saúde' descritos por artigos desta categoria

Sobral, A. et al., (2010); Sebek, K. et al., (2014); Howland, R. E. et

- Sistemas estruturados (p. e. SIM, Sinan, SIH, S2ID, EM-DAT);

al., (2014); Xavier, D. R. et al., (2014); Minervino, A. C., \& Duarte, E.

C. (2016); Rufino, R. et al., (2016)
- Sistema de informação não estruturado (p. e. blog, postagens e mídia eletrô-

nica);

- Sistema de informações georreferenciadas;

- Instituições produtoras e/ou disseminadoras de informações (p. e. Instituto Brasileiro de Geografia e Estatística - IBGE; Instituto de Pesquisa Econômica Aplicada - Ipea);

- Sistemas de informação utilizados em Nova York (Sistema eletrônico de informações da atenção primária de Nova York, Sistema de rastreamento da Cruz Vermelha e Sistema eletrônico de registros de óbitos de Nova York).

Fonte: Elaboração própria1-69.

Os 43 artigos selecionados foram publicados entre os anos de 1980 e 2017. Desse total, $6,98 \%$ (3) foram publicados na década de 1980 , com abordagem voltada para os efeitos dos desastres sobre a saúde (1) e para as ações de saúde após desastres (2). Durante a década de 1990 , foram publicados $23,26 \%$ dos artigos (10). Os pontos centrais abordados seguiram o perfil verificado na década anterior, a saber: efeitos dos desastres sobre a saúde (8); ações do setor saúde (1); ou uma combinação das duas temáticas (1). O mesmo ocorreu na primeira década dos anos 2000 , período no qual foram publicados 6,98\% (3) dos artigos, que abordaram as temáticas discutidas nas décadas 1980 e 1990. Todos os artigos das três décadas foram publicados no Portal BVS.

A partir de 2010, houve a ampliação tanto da abordagem quanto do número de publicações sobre desastre e vigilância em saúde. Dos artigos, $62,79 \%$ (27) foram publicados nessa década, e passaram a contar com discussões que tratavam, além dos efeitos dos desastres sobre a saúde e das ações do setor saúde, também as condições de vulnerabilidade em desastre (3) e o sistema/fluxo de informações 
(6) como assuntos centrais. A partir de 2010, foram verificadas 3 publicações no portal BVS, 12 no portal Capes e 14 no PubMed.

Na plataforma PubMed, 85,71\% (12) estavam relacionados com os efeitos dos desastres sobre a saúde, com as ações do setor saúde em desastres ou abordavam a combinação dos dois temas. Padrão semelhante foi observado no portal BVS, em que $94,12 \%$ (16) das publicações abordavam uma dessas temáticas ou a combinação delas. No portal Capes, $50 \%$ (6) das publicações eram sobre os efeitos dos desastres na saúde, sendo que uma abordava essa temática de forma conjunta ao sistema/fluxo de informação em saúde e outra abordava em conjunto com as ações do setor saúde em desastres.

Os artigos sobre sistema/fluxo de informação em saúde e condições de vulnerabilidade e desastres apareceram em menor número e foram publicados a partir de 2010. No portal BVS, apenas 5,88\% dos artigos (1) se referiram ao sistema/fluxo de informação em saúde, enquanto na PubMed, esse percentual correspondeu a $14,29 \%$ (2); e no portal Capes, a $25 \%$ (3). A categoria condições de vulnerabilidade e desastres foi tema central em $25 \%$ (3) dos artigos do portal Capes. O portal BVS apresentou um artigo dessa categoria que, por estar publicado também pelo portal Capes, foi considerado na pesquisa apenas como integrante deste último para evitar duplicidades. A base de dados PubMed não apresentou publicações contendo a temática de vulnerabilidade.

\section{Sistemas/fluxo de informação em saúde}

Neste tópico, será abordado o sistema de informação considerando-o como um conjunto de arquivos, incluindo os procedimentos para armazenamento, que permitem a manipulação e a recuperação de informações, sendo o fluxo destas parte integrante deste processo ${ }^{\mathbf{1 2}}$.

A maior parte dos estudos desta categoria era descritiva, com foco na comparação dos dados obtidos de diferentes bases nacionais e internacionais relacionadas com agravos à saúde ou danos dos desastres ${ }^{13,14}$. A construção de um banco de dados a partir de bases públicas já existentes para trazer novos apontamentos sobre a relação desastre e saúde também foi abordada ${ }^{15}$, enquanto outros temas giraram em torno da construção e implementação de sistemas de informação e/ou sistemas de vigilância em desastres de origem natural ${ }^{10}$. Entre os sistemas de informação utilizados em situação de desastres citados pela literatura, incluem-se sistemas nacionais e internacionais, como: os sistemas de informação em saúde brasileiros - SIS (por exemplo, Sistema de Informação sobre Mortalidade - SIM; Sistema de Informação de Agravos de Nnotificação - Sinan; Sistema de Informações Hospitalar - SIH, Sistema de Informação da Atenção Básica - Siab); Sistema Integrado de Informações sobre Desastres - S2ID; do Instituto Brasileiro de Geografia e Estatística - IBGE; o banco de dados internacional sobre desastres - Emergency Events Database - EM-DAT; o sistema eletrônico de registros de óbitos de Nova York e o sistema eletrônico de informações da atenção primaria em Nova York ${ }^{10,13-17}$.

Os estudos, em geral, ressaltam também a utilização simultânea de mais de um sistema de informação para monitorização de necessidades e condições de saúde em desastres, que englobam os vários níveis de atenção, primária, secundária e terciária.

Apesar de as discussões sobre o sistema/ fluxo de informação de saúde em casos de desastres serem mais recentes (quadro 1), sua importância é relatada em artigos publicados há décadas. De Ville de Goyet ${ }^{18}$, ao enfatizar o papel da vigilância e dos efeitos dos desastres sobre a saúde, apontava a necessidade de obter informações rápidas e confiáveis que pudessem auxiliar na tomada de decisão por parte de gestores. Nesse sentido, a importância da informação ganha destaque, uma vez que serve de embasamento tanto para a execução de atividades operacionais, gerenciais e estratégicas, quanto para a tomada de decisões em vários níveis hierárquicos das organizações ${ }^{19}$. 
As implicações relativas a falhas no fluxo de informação, como a destinação equivocada de suprimentos ou socorristas, também foram verificadas durante esta revisão $0^{20}$. Os sistemas de informação são pautados nos dados, portanto, a qualidade desses está diretamente relacionada com melhora dos registros. Corroborando essa ideia, o Marco de Sendai, ao abordar a compreensão do risco, enfatiza a necessidade de melhorar as ferramentas de medição, coleta, análise e divulgação de dados na gestão do risco de um desastre?.

A importância das informações foi abordada em textos que integraram esta revisão, como de Rufino et al..$^{13} \mathrm{e}$ Xavier et al..$^{15}$, ao salientarem que elas são ferramentas para análises de condições sociais, de saúde, ambientais, de exposição e vulnerabilidades que auxiliam na determinação do risco ao qual a população está exposta, podendo orientar ações de mitigação ou eliminação dos efeitos dos desastres sobre a saúde.

Nacionalmente, o principal sistema de informação sobre desastres é chamado S2ID, produto da Secretaria Nacional de Proteção e Defesa Civil, em que são encontradas informações sobre o número de eventos e a gestão de risco de desastres no Brasil. Esse sistema tem passado por constante aperfeiçoamento desde sua criação, em 2012, e teve suas limitações e potencialidades discutidas em trabalhos integrantes desta revisão $0^{10,14,15}$. Para Xavier et al. ${ }^{15}$, a melhoria do S2ID tem contribuído para sua dinamicidade, porém, o preenchimento inadequado de formulários que o alimentam ainda é uma limitação, o que colabora para a baixa qualidade dos registros e dificulta a utilização dessas informações para análises de saúde. Outras limitações dos sistemas de informações que registram desastres foram apontadas por Sobral et al. ${ }^{10}$ e Minervino et al. ${ }^{14}$ e incluem: i) a dissemelhança dos critérios para inclusão do evento em diferentes sistemas de informação (por exemplo, S2ID e EM-DAT); ii) as estimativas de danos em decorrência de um desastre, que podem não ser muito acuradas, já que os formulários do S2ID não têm esse foco e precisam ser produzidos em um curto espaço de tempo após o evento; iii) e a influência subjetiva por parte de gestores municipais e estaduais nas estimativas de danos a fim de obter mais recursos externos.

Limitações nesses sistemas também podem decorrer não apenas de fragilidades no momento do registro ou do tipo de informação gerada, como mencionado acima para o S2ID, mas também na esfera político-administrativa. Cortes de investimentos para a realização do recenciamento demográfico brasileiro previsto para 2020, por exemplo, poderão impactar o número de variáveis pesquisadas e, consequentemente, ter efeitos diretos nos bancos de dados do IBGE ${ }^{21}$. Ainda, muitos são os desafios vivenciados no SIS, como: i) as várias subdivisões de software e sistemas, sem interligação, que dificultam uma visão sistêmica, ii) a dificuldade em criar padrões para a interoperabilidade das informações, em virtude das heterogeneidades existentes em países em desenvolvimento; e iii) o aperfeiçoamento de tecnologias que exigem a capacitação dos profissionais para aplicá-las (como os prontuários eletrônicos dos pacientes) ${ }^{22,23}$.

Contudo, destaca-se que a inclusão de novas tecnologias da informação e comunicação tem muito a contribuir para a gestão do risco de um desastre, a exemplo de sua utilização no terremoto do Haiti, onde ferramentas de crowdsourcing permitiram o acesso a um maior número de informações e colaboraram para organização de mapas e solicitação de demandas de atendimento ${ }^{15,24}$.

Outros exemplos da utilização dos sistemas de informação para análises de saúde verificados entre os estudos analisados neste trabalho foram citados por Sebek et al. ${ }^{16}$ e Howland et al. ${ }^{17}$. No primeiro, dados do sistema de informação da atenção primária em Nova York foram utilizados para avaliar os impactos do furacão Sandy na saúde pública, visando ações de resposta e recuperação a desastres. No segundo, dados do sistema eletrônico de registro de óbito do departamento de saúde de Nova York foram utilizados pela cruz vermelha americana para realizar o rastreamento 
dos óbitos decorrentes do furacão Sandy. Na ocasião, a cruz vermelha conseguiu capturar a circunstância dos óbitos além de verificar, por exemplo, que o impacto da tempestade estava sendo subestimado por pessoas preocupadas em proteger suas propriedades ${ }^{\mathbf{1 7}}$.

Dessa forma, os estudos relataram que a utilização dos sistemas de informação integrados pode auxiliar na interação das equipes e na coordenação de atendimentos, além de serem importantes em análises sobre a frequência e intensidade dos desastres e sua relação com outros eventos como saúde, mudanças climáticas e condições sociais, facilitando e ampliando possibilidades de análise da realidade para compreensão das causas e efeitos de um desastre ${ }^{10,14,15}$.

Diante do exposto, considera-se que o sistema/fluxo de informações brasileiro possui muitos desafios a serem superados, não obstante as várias limitações descritas anteriormente, e que a elaboração de um sistema de informações integrado de forma transdisciplinar pode fornecer inúmeras possibilidades de análises e tem muito a contribuir para a gestão do risco de desastres no Brasil.

\section{Condições de vulnerabilidade e desastres}

Nesta categoria, os artigos abordaram a análise das vulnerabilidades socioambientais em populações atingidas por desastres e sua relação com estratégias da gestão do risco ${ }^{\mathbf{2 5}, \mathbf{2 6}}$. Outra abordagem presente foi o desenvolvimento de um índice socioambiental com o intuito de predizer a vulnerabilidade a desastres de origem natural no estado do Rio de Janeiro ${ }^{27}$.

O risco de um desastre existe a partir de uma sobreposição de condicionantes composta por uma ameaça (natural, tecnológica ou antrópica), uma população exposta/vulnerável e baixa capacidade adaptativa, que funciona como um catalisador das fragilidades locais. Nesse sentido, o risco se desenvolve a partir de uma construção social que integra processos relacionados com o modelo de desenvolvimento econômico, ambiental e de proteção social presentes em uma localidade $5,28,29$.

As condições de vulnerabilidade são responsáveis por influenciar tanto a exposição, potencializando a predisposição de uma determinada localidade a sofrer os impactos dos desastres, como a capacidade adaptativa, por meio de limitações em atividades de prevenção, mitigação, preparação, reposta e recuperação em localidades afetadas 18,25-28,30-32.

No estudo que integrou esta revisão e que analisou os terremotos que atingiram o Chile em 2010 e o Haiti em 2011, verificou-se que o primeiro atingiu uma maior magnitude na escala Ricther, porém, o número de vítimas fatais chegou a ser 519 vezes maior no segundo devido às condições de vulnerabilidade socioambiental mais acentuadas no Haiti'26. Dessa forma, na ocasião de um desastre de origem natural, as vulnerabilidades socioambientais são evidenciadas e se materializam na forma de consequências mais graves para determinados grupos ${ }^{33}$.

Os estudos analisados nesta revisão salientam que os desastres não só afetam com maior intensidade populações mais pobres como também comprometem condições essenciais para sobrevivência, como disponibilidade de água e alimentos, elevando ainda mais o risco de agravos de saúde que pode resultar em uma cronificação do desastre ${ }^{\mathbf{2 5}, \mathbf{2 6}}$. Ratificando essa ideia, destacam-se os ciclones Idai e Kenneth que atingiram Moçambique em 2019 e afetaram mais de 1 milhão de pessoas. Essa localidade, mesmo antes dos ciclones, já enfrentava obstáculos para os cuidados em saúde e infraestrutura em meio a sérias condições de pobreza e insegurança alimentar; após os ciclones, passou a enfrentar simultaneamente outros problemas como o aumento do número de casos de cólera ${ }^{34}$.

Levando em consideração a importância de conhecer a vulnerabilidade das populações, muitos esforços têm sido despendidos com o intuito de melhorar a compreensão dessa temática, como pode ser verificado no trabalho que integrou este estudo, em que Guimarães 
et al. ${ }^{27}$ abordaram a construção de um índice de vulnerabilidade socioambiental para predizer a vulnerabilidade de uma população à ocorrência de desastres de origem natural no estado do Rio de Janeiro.

Outras metodologias verificadas nesta revisão incluem estudos de caso, como o apresentado por Freire et al. ${ }^{25}$, que avaliou a relação entre a vulnerabilidade e a ocorrência de inundações em Alagoas no ano de 2010, e concluiu que, mesmo entre os vulneráveis, existiam grupos ainda mais susceptíveis que enfrentavam a propagação do ciclo da pobreza e a cronificação do desastre; e o estudo de Freitas et al. ${ }^{26}$ que comparou os terremotos do Haiti e as chuvas da região Serrana do estado do Rio de Janeiro e observou que tanto a gestão do risco de um desastre quanto a resiliência estão intrinsicamente associadas às vulnerabilidades da população e devem ser consideradas em seu contexto, pois implicam medidas a serem desenvolvidas em um processo longo que envolve o desenvolvimento econômico e social de uma localidade sob uma perspectiva sustentável26.

Reafirmando o olhar da gestão de risco na perspectiva do desenvolvimento sustentável, a Agenda de 2030 propõe os Objetivos de Desenvolvimento Sustentável (ODS) que podem contribuir para a redução de risco dos desastres, incluindo questões como acabar com a pobreza, com a fome, e garantir a segurança alimentar, ratificando sua importância para o desenvolvimento humano ${ }^{8}$.

Além disso, as projeções da mudança do clima para as próximas décadas sugerem um aumento na intensidade e frequência de fenômenos naturais que podem interferir diretamente no alcance de objetivos como o da erradicação da pobreza ${ }^{\mathbf{2}, \mathbf{8}}$. Nessa perspectiva, destaca-se a importância do Marco de Sendai que, a exemplo dos ODS, estipulou metas a serem alcançadas até 2030 (por exemplo, redução da mortalidade por desastres, diminuição das perdas econômicas), e pode auxiliar no fortalecimento da governança do risco de desastre, na organização de setores públicos e privados em busca de parcerias dentro do escopo da resiliência ${ }^{7,8}$.

Por fim, ressalta-se que, no Brasil, os riscos associados a desastres possuem estreita relação com as já descritas condições de vulnerabilidade socioeconômicas, ambientais e culturais, além disso, as desigualdades regionais, muitas vezes, esbarram em estruturas de enfrentamento fragilizadas no que se diz respeito à forma de lidar com questões ambientais, sociais e de governança, que interferem diretamente na capacidade adaptativa das localidades diante de um desastre ${ }^{29}$.

\section{Efeito dos desastres na saúde}

Os efeitos sobre a saúde das populações são largamente descritos pela literatura; dos 43 artigos, 28 têm essa temática como ponto central. São discutidas morbimortalidades relacionadas com doenças transmissíveis e não transmissíveis, em curto, médio e longo prazo após desastres (quadro 1) 13,18,30,31,35-58.

A relação entre a precipitação, enchentes, inundações, precárias condições de saneamento e a transmissão da leptospirose - e de outras doenças de veiculação hídrica também - foi verificada entre os artigos desta revisão ${ }^{\mathbf{4 3}}$. Validando essa ideia, estudos têm demonstrado que as médias mensais de precipitação podem se tornar aliadas aos planejamentos de saúde, inclusive para ações de vigilância, por serem um forte fator relacionado com o aumento do número de casos da doença ${ }^{59,60}$.

De modo geral, doenças de veiculação hídrica e transmitidas por vetores (como cólera, dengue, malária) têm seu padrão de ocorrências diretamente impactado por desastres, principalmente em lugares com piores condições socioeconômicas. Entre os artigos que integraram esta revisão, essa realidade foi descrita, por exemplo, no estudo que abordou o aumento do número de casos de algumas dessas doenças na Guatemala, na Nicarágua, em Honduras e em El Salvador após o furacão Mitch, que atingiu a América Central em $1998^{30}$. 
Sob outra perspectiva, os trabalhos de Duff et al. ${ }^{35,36,55}$, também integrantes desta revisão, evidenciaram que, após o furacão Gilbert na Jamaica em 1988, a dieta materna, que comumente continha uma quantidade adequada de produtos frescos (bananas maduras, laranja, vegetais) teve de ser alterada, visto que o furacão causou grande impacto sobre as colheitas daquele ano. Com isso, a ingestão de folato no período periconcepcional diminuiu, e isso pode ter levado ao aumento de nascidos vivos com defeitos do tubo neural após o furacão.

É interessante destacar que, independentemente da etiologia, os desastres interferem na dinâmica das doenças em um território. Condições de seca e estiagem podem prejudicar a qualidade da água disponível para o consumo humano e gerar impactos sobre a saúde que englobam tanto doenças de veiculação hídrica quanto transmitidas por vetores $\mathbf{1 3 , 4 9 , 5 0}$. Além disso, podem prejudicar a disponibilidade e o acesso a alimentos, levando a impactos sobre o estado nutricional dos afetados 18,30,49-51.

Outro estudo que integrou esta revisão relatou também a associação entre desastres e doenças respiratórias, ao evidenciar que, na Amazônia, em 2005 e 2010, houve aumento significativo de internações por doenças respiratórias em menores de cinco anos que residiam em municípios muito expostos à seca. Vale ressaltar que, nesse período, também ocorreu aumento do número de queimadas, o que prejudicou ainda mais a qualidade do ar e o estado respiratório dos indivíduos, já que o número de partículas nocivas suspensas no ar aumentou ${ }^{31}$.

A ocorrência de doenças respiratória é frequentemente descrita dentro dos impactos à saúde decorrente dos desastres devido, por exemplo, à baixa umidade do ar, à poeira e ao calor presentes em períodos de seca e estiagem, à exposição a partículas nocivas durante a limpeza de áreas atingidas por inundações ou mesmo pela exposição a doenças transmitidas por gotículas e aerossóis em ambientes superlotados após desastres

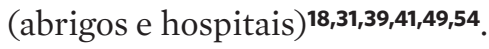

$\mathrm{O}$ adoecimento mental também foi abordado em artigos que integraram esta revisão, por exemplo, no estudo que avaliou os acontecimentos sequentes à tempestade Xyinthia, na França em 2010. Cerca de 5 mil casas foram inundadas, o que suscitou a necessidade de intervenções psicológicas prestadas pela unidade de emergência médico-psicológica do departamento de Charente-Maritime. Foi constatado que o pico de maior incidência de casos ocorreu no quarto mês após o evento, período que coincidiu com a realocação de vítimas que se encontravam em residências temporárias e com a visita dos especialistas para avaliar o valor das casas inabitáveis após a inundação. Os sintomas psicológicos mais frequentes foram sinais de depressão e de ansiedade; quase todos os pacientes com sinais psicológicos precisavam de acompanhamento médico após o evento, ratificando a necessidade de acompanhamento de saúde também em médio e longo prazo após desastres ${ }^{40}$.

Outros problemas de saúde pública relacionados com os desastres, além de doenças mentais, incluem: lesões, mortes e migração. Estes muitas vezes estão diretamente relacionados entre si, pois, além de serem retirados das suas residências, os sobreviventes de desastre são obrigados a conviver com a perda de familiares próximos e/ou com lesões físicas e emocionais por toda a vida. Na década de 1980, vários estudos apontavam a presença de transtornos mentais em crianças e adultos que haviam passado por situações de desastres ${ }^{53,58}$. Atualmente, sabe-se que essas situações podem acarretar problemas de saúde em curto, médio e longo prazo, que incluem: transtorno do estresse pós-traumático, depressão, ideação suicida e distúrbios do sono $\mathbf{4 0 , 4 7 , 4 9 , 5 0 , 5 3 , 5 8 , 6 1}$.

Dessa forma, os efeitos dos desastres naturais sobre a saúde constituem um grande desafio à saúde pública, possuem etiologia multifatorial e decorrem de uma série de fatores que envolvem a vulnerabilidade populacional, o modelo de desenvolvimento econômico e a mudança do clima. 


\section{Ações do setor saúde em desastres}

Dos 43 artigos, em 13 esta discussão teve papel central - muitas vezes associados aos efeitos dos desastres sobre a saúde -, sendo o primeiro de 1983, o que denota a preocupação antiga do setor por essa temática (quadro 1) $32,38-41,47,49,54,62-67$

De forma geral, percebe-se que as ações de saúde foram otimizadas e direcionadas ao longo dos anos, evoluindo conjuntamente à temática de desastres, e passaram a abarcar a noção de que um evento não ocorre de forma dissociada do quadro social. Apesar disso, as ações direcionadas ao manejo e recuperação, muitas vezes, sobressaem-se à nova lógica, que tem como ponto substancial a redução do risco, ou seja, a prevenção, a mitigação e a preparação para um desastre $32,38-41,47,49,54,62-68$.

As ações de mitigação evitam ou minimizam os prejuízos causados pelo desastre sobre as populações, o ambiente e os serviços essenciais - isto é, abastecimento de água, energia e transporte. Além disso, a cada dólar que é investido em atividades de prevenção, economiza-se aproximadamente sete dólares que seriam gastos com a recuperação após um desastre ${ }^{68}$.

Considerando a natureza interdisciplinar e intersetorial do risco, a inserção do setor saúde nas estratégias nacionais para a redução do risco trazem inúmeros benefícios, como a diminuição de possíveis gastos após um evento (por exemplo, pela redução do número de adoecimentos, feridos e mortos) e o planejamento e treinamento de pessoal, fundamentais para ações mais efetivas em grandes desastres ${ }^{\mathbf{1 8 4 4}}$. Cabe destacar ainda a importância das ações da vigilância em saúde (ou seja, epidemiológica, ambiental, sanitária e saúde do trabalhador), essenciais para o aumento da capacidade de resposta, sendo seu desempenho pré-desastre determinante também para sua eficiência após o evento ${ }^{\mathbf{4 3}, 67}$.

Corroborando a ideia de interdisciplinaridade, o Marco de Sendai ressalta a necessidade de trabalhar de forma articulada os desastres e as emergências de saúde pública, em substituição aos modelos fragmentados de governança dos riscos. Tal fato foi evidenciado no estudo realizado por Freitas et al.69, ao discutir os desafios para a saúde coletiva e o SUS, em uma reflexão sobre os desastres do rompimento de barragem ocorridos em Mariana e Brumadinho, Minas Gerais.

A redução de riscos é uma das ações essenciais da saúde pública. Entre a literatura selecionada, Grigoletto et al. ${ }^{49}$, em seu estudo sobre a gestão das ações do setor saúde, evidenciam algumas contribuições desse setor no período pré-desastre, como: i) participação na elaboração de planos de contingência e resposta a emergências; ii) implementação e fortalecimento de comitês de saúde em desastres; iii) articulação intersetorial e interinstitucional, local e regional; iv) ações de capacitação e treinamento tanto de equipes assistenciais quanto de vigilância ${ }^{49,66}$. Outras ações também foram apontadas por Roces et al.56, a saber, o fortalecimento da autossuficiência das comunidades, a educação em primeiros socorros e noções de salvamento básico para a comunidade.

Contudo, ressalta-se que o setor saúde ainda possui um longo caminho na atuação em redução de risco e resiliência, e que este inclui vários desafios, por exemplo, sua própria vulnerabilidade, expressa pela falta de preparação dos profissionais e instituições ou pelas unidades localizadas em áreas de risco ${ }^{26}$.

Em relação às ações de recuperação e resposta após os eventos, os textos selecionados permitem o compartilhamento das lições aprendidas e das práticas utilizadas para mitigação dos impactos dos desastres, fazendo isso a partir de contextos distintos de desenvolvimento social, econômico e capacidade adaptativa, uma vez que descrevem as ações adotadas após desastres em diferentes localidades.

Como exemplo, pode-se citar o estudo de Williams et al. ${ }^{39}$, que leva a reflexões sobre medidas tanto de preparação quanto de resposta, ao evidenciar as ações adotadas antes e após o furacão Ivan na Jamaica em 2004. 
Neste, é relatado que o hospital universitário em questão contava com consultores de medicina de emergência em tempo integral, e são sugeridas ações a serem implementadas para preparação em grandes eventos, como:

- Os profissionais do departamento de emergência devem estar no estabelecimento de saúde antes do evento, caso haja interrupção de vias;

- Estar preparado para situações nas quais haja interrupção do abastecimento de água, principalmente quando feridas e lacerações são prováveis;

- Ter um plano para operar equipamentos que necessitam de energia elétrica, caso o fornecimento seja interrompido etc.

Apisarnthanaraka et al. ${ }^{41}$, por sua vez, apresentam contribuições sobre a importância da limpeza e da descontaminação de unidades de saúde após inundações e também de ações de vigilância, como análises de amostras de água e ar por pelo menos 12 meses pós-evento. $\mathrm{O}$ estudo apontou evidencias que sugerem $\mathrm{o}$ aumento das infecções relacionadas com os cuidados de saúde, após as inundações por águas contaminadas por resíduos humanos ou animais, relativas à contaminação de equipamentos e infraestruturas de cuidados de saúde. Ações de vigilância foram citadas também por Ridpath et al.63, no estudo que abordou os desafios à implementação da vigilância de doenças transmissíveis em abrigos de evacuação após o furacão Sandy, em Nova York. Esse trabalho descreve ações de vigilância como: i) contato telefônico com médicos; ii) visitas diárias aos abrigos; e iii) treinamento da equipe médica sobre o sistema de vigilância e de funcionários sobre epidemiologia.

Cabe aqui destacar que o sucesso das ações de um sistema de vigilância está intrinsecamente relacionado com a existência prévia desse serviço, fato este já apontado no estudo de Iversson em 199167, que integrou esta pesquisa, ao abordar a temática vigilância em saúde e desastres. Após quase três décadas, essa reflexão ainda é atual e exemplifica as grandes dificuldades vivenciadas pela vigilância em saúde, especificamente relacionadas com desastres ao longo da história. O referido estudo traz contribuições também sobre a necessidade de realizar a vigilância epidemiológica em longo prazo no período pós-desastre, justificada pelas alterações ambientais que podem ter sido causadas pelo evento, assim como pela possibilidade de circulação de novos agentes inexistentes até então, ou ainda pelas perdas econômicas e de qualidade de vida que geram consequências sobre a saúde.

Por fim, ressalta-se que o fortalecimento da saúde no contexto da gestão do risco de desastre perpassa ações de prevenção, mitigação, preparação, resposta e recuperação; incluem abordagens que devem contemplar instalações seguras, profissionais treinados, diagnóstico de realidade, planejamento estratégico, acompanhamento de saúde a afetados direta e indiretamente por desastres, em curto, médio e longo prazo, além de apoio político/ social aos vulneráveis em busca da resiliência populacional e institucional.

\section{Considerações finais}

O envolvimento da vigilância em saúde na gestão do risco de um desastre deve ocorrer de forma coordenada com os vários níveis de assistência à saúde, a fim de diminuir e controlar os impactos dos desastres sobre a saúde pública. Dessa forma, estimulam-se investigações que apontem mais claramente as experiências e desafios relacionados com a atuação intrassetorial e intersetorial da vigilância.

Ademais, não foram verificados artigos que abordassem, em profundidade, a temática da troca de informações entre as instituições envolvidas em um desastre, apesar da função substancial desse processo para ações de gestão de risco. A maioria dos artigos 
revisados abordou apenas um desastre específico, salientando a necessidade de trabalhos que discutam a noção da combinação de desastres de origem natural com os de origem tecnológica e vice-versa.

Por último, ressalta-se a necessidade de esforços para diminuição das vulnerabilidades do próprio setor saúde com o intuito de aperfeiçoar suas ações em favor das comunidades e a necessidade de mudança gradativa do perfil de investimentos no Brasil, refletida também na necessidade de maior articulação entre níveis federal, estadual e municipal nesse processo.

\section{Agradecimentos}

Ao Instituto Nacional de Ciência e Tecnologia para Mudanças Climáticas e à Fundação de Amparo à Pesquisa de Minas Gerais (Fapemig).

\section{Colaboradores}

Santos R (0000-0002-8999-2143)* contribuiu em todas as etapas de elaboração do artigo. Menezes JA (0000-0002-1032-1997)* contribuiu para elaboração do conteúdo e revisão final do arquivo. Souza CM (0000-0003-42511004)* contribuiu para elaboração do conteúdo e revisão final do arquivo. Confalonieri U (0000-0002-8589-2866)* contribuiu para concepção e planejamento do arquivo. Freitas CM (0000-0001-6626-9908)* contribuiu para concepção, planejamento e participou da revisão final do artigo.

\section{Referências}

1. Intergovernmental Panel on Climate Change. Summary for Policymakers. Climate Change and Land: an IPCC special report on climate change, desertification, land degradation, sustainable land management, food security, and greenhouse gas fluxes in terrestrial ecosystems [internet]. Geneva: IPCC; WMO; Unep; 2019. [acesso em 2019 ago 20]. Disponível em: https://www.ipcc.ch/srccl/chapter/summary-for-policymakers/.
2. Intergovernmental Panel on Climate Change. Climate Change 2014: Synthesis Report. A Report of the intergovernamental panel on Climate Change [internet]. Geneva: IPCC WMO Unep; 2014. [acesso em 2016 set 23]. Disponível em: http://www.ipcc.ch/pdf/assessment-report/ar5/syr/SYR_AR5_FINAL_full_wcover. pdf.

3. Universidade Federal de Santa Catarina; Centro de Estudos e Pesquisas em Engenharia e Defesa Civil.
*Orcid (Open Researcher and Contributor ID). 
Atlas Brasileiro de Desastres Naturais 1991 a 2012. Florianópolis: CEPED; UFSC; 2013.

4. Organización de las Naciones Unidas. Estrategia Internacional para la Redución de Desastres. Rev EIRD Informa [internet]. 1999 [acesso em 2017 fev 20]; (14). Disponível em: http://www.eird.org/esp/revista/ No14_99/index_14e.htm.

5. Narváez L, Lavell A, Ortega GP. La gestión del riesgo de desastres: un enfoque basado en procesos. Lima: Secretaria general de la Comunidad Andina; 2009.

6. Brasil. Ministério da saúde. Guia de preparação e resposta às inundações para a gestão municipal do Sistema Único de Saúde. Brasil, DF: Ministério da saúde; 2011.

7. Um office for Disaster Risk Reduction. Sendai Framework for Disaster Risk Reduction 2015-2030 [internet]. Geneva: United Nations; 2015. [acesso em 2017 fev 21]. Disponível em: http://www.preventionweb.net/files/43291_sendaiframeworkfordrren.pdf.

8. Um office for Disaster Risk Reduction. Disaster risk reduction and resilience in the 2030 agenda for sustainable development [internet]. 2015. [acesso em 2017 fev 21]. Disponível em: https://www.unisdr.org/ files/46052_disasterriskreductioninthe2030agend. pdf.

9. Brasil. Ministério da Saúde, Vigilância em Saúde. Portal da saúde [internet]. 2017. [acesso em 2019 ago 20]. Disponível em: http://www.saude.gov.br/vigilancia-em-saude/sobre-vigilancia-em-saude.

10. Sobral A, Freitas CM, Andrade EV, et al. Desastres naturais - sistemas de informação e vigilância: uma revisão da literatura. Epidemiol. serv. saúde [internet]. 2010; 19(4):389-402. Disponível em: http://dx.doi. org/10.5123/S1679-49742010000400009.

11. Rocha V, Ximenes EF, Carvalho ML, et al. O tema desastre na área da saúde: Perfil da produção técnico-científica na base de dados especializada em desastres da biblioteca virtual em saúde (BVS). Ciênc. Saúde Colet. 2014; 19(9):3775-90.
12. Centro Latino-Americano e do Caribe de Informação em Ciências da Saúde. Descritores em Ciências da Saúde (DeCS) [internet]. 2019. [acesso em 2017 fev 21]. Disponível em: http://decs.bvs.br/.

13. Rufino R, Gracie R, Sena A, et al. Diarrhea outbreaks in northeastern Brazil in 2013, according to media and health information systems - Surveillance of climate risk and health emergencies. Ciênc. Saúde Colet. 2016; 21(3):777-8.

14. Minervino AC, Duarte EC. Loss and damage affecting the public health sector and society resulting from flooding and flash floods in Brazil between 2010 and 2014 - Based on data from national and global information systems. Ciênc. Saúde Colet. 2016; 21(3):68594.

15. Xavier DR, Barcellos C, Barros HS, et al. Organização, disponibilização e possibilidades de análise de dados sobre desastres de origem climática e seus impactos sobre a saúde no Brasil. Ciênc. Saúde Colet. [internet]. 2014 [acesso em 2018 jan 5]; 19(9):365768. Disponível em: http://dx.doi.org/10.1590/141381232014199.00992014 .

16. Sebek K, Jacobson L, Wang J, et al. Assessing capacity and disease burden in a virtual network of New York city primary care providers following hurricane sandy. J Urban Health. 2014; 91(4):615-22.

17. Howland RE, Madsen AM, Nicaj L, et al. Assessing electronic death registration and american red cross systems for mortality surveillance during hurricane sandy, October 29-November 10, 2012, New York City. Disaster Med Public Health Prep. 2014; 8(6):489-91.

18. De Ville GC. Las enermedades transmisibles y la vigilancia epidemiologica en situaciones de desastres naturales. Bol la Of Sanit Panam. 1980; 89(4):353-9.

19. Damasceno VG, Gonçalves IR, Lima WD. A importância da informação nas organizações. Rev Tecnol em Projeção. 2018; 9(2):78-90.

20. Ludwig L, Mattedi MA, Firmino RJ, et al. As tecnologias da informação e comunicação na gestão dos 
riscos de desastres: o caso do Alerta em Blumenau/ SC. Rev Tecnol e Soc. 2018; 14(31).

21. Estadão conteúdo. Após definir cortes, IBGE propõe reduzir questões do Censo 2020 [internet]. Exame. 2019 maio 28. [acesso em 2018 jan 5]. Disponível em: https://exame.abril.com.br/brasil/apos-definir-cortes-ibge-propoe-reduzir-questoes-do-censo-2020/.

22. Bittar OJN, Biczyk M, Serinolli MI, et al. Sistemas de informação em saúde e sua complexidade. Rev Adm em Saúde. 2018; 18(70):1-18.

23. Fornazin M, Joia LA. Articulando perspectivas teóricas para analisar a informática em saúde no Brasil. Saúde e Soc. 2015; 24(1):46-60.

24. Margoto JB. Usos e aplicações de novas TIC'S na gestão de desastres naturais. Uses and applications of new ICTs in natural disaster management. Perspect em Ciênc. Info. [internet]. 2017 [acesso em 2018 jan 5]; 22(3):3-15. Disponível em: http://portaldeperiodicos.eci.ufmg.br/index.php/pci/article/view/2534/0.

25. Freire NCF, Bonfim CV, Natenzon CE. Vulnerabilidade socioambiental, inundações e repercussões na saúde em regiões periféricas: O caso de Alagoas, Brasil. Ciênc. Saúde Colet. 2014; 19(9):3755-62.

26. Freitas CM, Carvalho ML, Ximenes EF, et al. Vulnerabilidade socioambiental, redução de riscos de desastres e construção da resiliência - lições do terremoto no Haiti e das chuvas fortes na região serrana, Brasil. Ciênc. Saúde Colet. 2012; 17(6):1577-86.

27. Guimarães RM, Mazoto ML, Martins RN, et al. Construção e validação de um índice de vulnerabilidade socioambiental para a vigilância e gestão de desastres naturais no estado do Rio de Janeiro, Brasil. Ciênc. Saúde Colet. 2014; 19(10):4157-65.

28. Organização Mundial da Saúde, Organização Pan-Americana da Saúde. Desastres Naturais e Saúde no Brasil. Brasília, DF: OMS; OPAS; 2015. [Série desenvolvimento Sustentável 2].

29. Almeida LQ, Welle T, Birkmann J. Disaster risk indi- cators in Brazil: A proposal based on the world risk index. Int J Disaster Risk Reduct [internet]. 2016 [acesso em 2018 jan 5]; 17:251-72. Disponível em: http:// dx.doi.org/10.1016/j.ijdrr.2016.04.007.

30. Pan American Health Organization; World Health Organization. Impact of hurricane mitch in Central America. Epid. Bulletin. [internet]. 1998 [acesso em 2018 jan 5]; 19(4). Disponível em: http://wwwl.paho. org/english/sha/epibul_95-98/be984mitch.htm.

31. Smith LT, Aragão LEOC, Sabel CE, et al. Drought impacts on children's respiratory health in the Brazilian Amazon. Sci Rep. 2014; 4:1-8.

32. Crane MA, Cho HG, Landrigan PJ. Implications of the world trade center health program (WTCHP) for the public health response to the great East Japan Earthquake. Ind Health. 2014; 52(1):5-12.

33. Cutter SL. A ciência da vulnerabilidade: modelos, métodos e indicadores. Rev Crit Cienc Sociais. 2011; (93):59-69.

34. eNCA. Cyclone Idai's death toll nears 1,000 in Mozambique, Zimbabwe [internet]. eNCA, DSCtv channel 403. 2019. [acesso em 2020 maio 3]. Disponível em: https://www.enca.com/news/cyclone-idais-death-toll-nears-1000-mozambique-zimbabwe.

35. Duff EMW, Copper ES. A post-hurricane Gilbert in Neural Tube Defects in Jamaica, associated with a diet comparatively low in folate, in the periconceptional period. West Indian Med J. 1992; (41):25.

36. Duff EMW, Cooper ES. Neural tube defects in Jamaica following Hurricane Gilbert. Am J Public Health. 1994; 84(3):473-6.

37. Malilay J, Real MG, Vanegas AR, et al. Public health surveillance after a volcanic eruption: Lessons from Cerro Negro, Nicaragua, 1992. Bull Pan Am Health Organ. 1996; 30(3):218-26.

38. Balluz L, Moll D, Martinez MGD, et al. Environmental pesticide exposure in Honduras following hurricane Mitch. Bull World Health Organ. 2001; 79(4):288-95. 
39. Williams EW, Williams-Johnson J, French S, et al. The effect of Hurricane Ivan on Emergency Department operations at the University Hospital of the West Indies. West Indian Med J. 2005; 54(4):232-5.

40. Raguenaud ME, Germonneau P, Leseigneur J, et al. Epidemiological surveillance linked to an outreach psychological support program after the Xynthia storm in Charente-Maritime, France, 2010. Prehosp Disaster Med. 2012; 27(5):483-8.

41. Apisarnthanarak A, Warren DK, Glen Mayhall C. Healthcare-associated infections and their prevention after extensive flooding. Curr Opin Infect Dis. 2013; 26(4):359-65

42. Goulding R, Jayasuriya N, Horan E. A Bayesian network model to assess the public health risk associated with wet weather sewer overflows discharging into waterways. Water Res [internet]. 2012 [acesso em 2018 jan 5]; 46(16):4933-40. Disponível em: http:// dx.doi.org/10.1016/j.watres.2012.03.044.

43. Guimarães RM, Cruz OG, Parreira VG, et al. Análise temporal da relação entre leptospirose e ocorrência de inundações por chuvas no município do Rio de Janeiro, Brasil, 2007-2012. Ciênc. Saúde Colet. 2014; 19(9):3683-92.

44. Freitas CM, Silva DRX, de Sena ARM, et al. Desastres naturais e saúde: Uma análise da situação do Brasil. Ciênc. Saúde Colet. 2014; 19(9):3645-56.

45. Pirard P, Goria S, Wakap SN, et al. No increase in drug dispensing for acute gastroenteritis after Storm Klaus, France 2009. J Water Health. 2015; 13(3):73745 .

46. Shrestha AC, Flower RLP, Seed CR, et al. Hepatitis E virus seroepidemiology: A post-earthquake study among blood donors in Nepal. BMC Infect Dis [internet]. 2016 [acesso em 2018 jan 5]; 16(1):1-9. Disponível em: http://dx.doi.org/10.1186/s12879-016-2043-8.

47. Sahni V, Scott AN, Beliveau M, et al. Public health surveillance response following the southern alberta floods, 2013. Can J Public Heal. 2016; 107(2):el42-8.
48. Ishiki A, Okinaga S, Tomita N, et al. Changes in cognitive functions in the elderly living in temporary housing after the great east Japan earthquake. PLos ONE [internet]. 2016 [acesso em 2018 jan 5]; 11(1):815. Disponível em: http://dx.doi.org/10.1371/journal. pone. 0147025 .

49. Grigoletto JC, Cabral AR, Bonfim CV, et al. Gestão das ações do setor saúde em situações de seca e estiagem. Ciênc. Saúde Colet. [internet]. 2016 Mar [acesso em 2018 jan 5]; 21(3):709-18. Disponível em: http://www. scielo.br/scielo.php?script=sci_arttext\&pid=S1413$-81232016000300709 \& \operatorname{lng}=\mathrm{pt} \& \mathrm{nrm}=\mathrm{iso} \& \mathrm{t} \operatorname{lng}=\mathrm{en}$.

50. Sena A, Freitas CM, Barcellos C, et al. Measuring the invisible: Analysis of the sustainable development goals in relation to populations exposed to drought. Ciênc. Saúde Colet. 2016; 21(3):671-83.

51. Alpino TA, Sena ARM, Freitas CM. Disasters related to droughts and public health - A review of the scientific literature. Ciênc. Saúde Colet. 2016; 21(3):80920.

52. Takahashi A, Ohira T, Uemura M, et al. Changes in Hepatobiliary Enzyme Abnormality after the Great East Japan Earthquake: The Fukushima Health Management Survey. Sci Rep [internet]. 2017 [acesso em 2018 jan 5]; 7(1):1-9. Disponível em: http://dx.doi. org/10.1038/s41598-017-00776-7.

53. Ahearn F. Ingresos en servicios de psiquiatría despues de un desastre natural. Bol la Of Sanit Panam. 1984; 97(4):325-35

54. Hospedales J, Lewis L, Lynch J, et al. Post-disaster Epidemiological Surveillance following Hurricane Hugo in Montserrat. West Indian Med J. 1989; 39(0043-3144):28.

55. Duff EMW, Cooper ES, Danbury CM, et al. Neural tube defects in hurricane aftermath. Lancet Public Health . 1991; 337:120-1.

56. Roces MC, White ME, Dayrit MM, et al. Risk factors for injuries due to the 1990 earthquake in Luzon, Philippines. Bull World Health Organ. 1992; 70(4):509-14. 
57. Armenian HK, Noji EK, Oganesian AP. A case-control study of injuries arising from the earthquake in Armenia, 1988. Bull World Health Organ. 1992; 70(2):251-7.

58. Lima BR, Chávez H, Samaniego N, et al. Trastornos psiquiátricos en víctimas de desastres en el ecuador. Bol Sanit Panam. 1992; 113(1):28-34.

59. Aleixo R, Neto JLS. Eventos pluviométricos extremos e saúde: perspectivas de interação pelos casos de leptospirose em ambiente urbano Hygeia. 2010; 6(11):118-32.

60. Soares TSM, Latorre MDRDDO, Laporta GZ, et al. Análise espacial e sazonal da leptospirose no município de São Paulo, SP, 1998 a 2006. Rev. saúde pública. 2010; 44(2):283-91.

61. Xavier DR, Barcellos C, Freitas CM. Eventos climáticos extremos e consequências sobre a saúde: o desastre de 2008 em Santa Catarina segundo diferentes fontes de informação. Ambient Soc. 2014; 17(4):273-94.

62. Peréz LJ. Misión de socorro d espues del terremoto en la lnõlia: un diario. Perspect Salud. 2001; (6):6-9.

63. Ridpath AD, Bregman B, Jones L, et al. Challenges to implementing communicable disease surveillance in New York city evacuation shelters after hurricane sandy, November 2012. Public health rep. 2014; 130(1):48-53.

64. Boyd AT, Cookson ST, Anderson M, et al. Centers for disease control and prevention public health response to humanitarian emergencies, 2007 - 2016. Emerg. infect. dis. 2017; 23(supl1):196-202.
65. Lemoine JF, Boncy J, Filler S, et al. Haiti's commitment to malaria elimination: Progress in the face of challenges, 2010-2016. Am. j. trop. med. hyg. 2017; 97(supl4):43-8.

66. Anon. Asistencia internacional y suministros médicos de urgencia después de desastres naturales. Desastr Prep y Mitigación -(OPS) [internet]. 1983 [acesso em 2018 jan 5]; (14). Disponível em: http://www. nzdl.org/gsdlmod?e=d-00000-00---off-0paho--000----0-10-0---0----0direct-10---4-------0-11--11-en-50--20-about---00-0-1-00-0--4----0-0-11-10-0utfZz-8$10 \& \mathrm{cl}=\mathrm{CL} 1.4 \& \mathrm{~d}=\mathrm{HASH01} \mathrm{cf134681e69384d1a421ad.}$ $1 \& g t=1$.

67. Iversson LB. Vigilância epidemiológica e controle de doença infecciosas pós-desastre. BIO. 1991; 3(1):67-9.

68. Liberato JA. Gastos em proteção e defesa civil para prevenção de desastres naturais versus resposta e reconstrução no brasil. Rev Técnica CNM. 2016 [acesso em 2018 jan 5]; (4):37-52. Disponível em: https:// www.cnm.org.br/cms/biblioteca_antiga/Gastos em proteção e defesa civil para prevenção de desastres naturais.pdf.

69. Freitas CM, Barcellos C, Asmus CIRF, et al. From samarco in mariana to vale in Brumadinho: Mining dam disasters and public health. Cad. Saúde Pública. 2019; 35(5):e00052519.

Recebido em 29/09/2019

Aprovado em 13/01/2020

Conflito de interesses: inexistente

Suporte financeiro: Instituto Nacional de Ciência e Tecnologia para Mudanças Climáticas, fase 2, sob concessão CNPq 465501

/ 2014-1. Fapesp concessão 2014 / 50848-9; Subsídio de Coordenação Nacional para Educação e Treinamento de Alto Nível (Capes) 16/2014. Fundação de Amparo à Pesquisa de Minas Gerais (Fapemig) 